\title{
Anomalous magnetic and dynamic behavior in magnetoresistive compounds: origin of bulk colossal magnetoresistivity
}

\author{
Vladimir Chechersky and Amar Nath \\ Department of Chemistry, Drexel University, Philadelphia, PA 19104, USA \\ E-mail: amar.nath@drexel.edu
}

Received January 2, 2002

\begin{abstract}
We present the results of our extensive Mössbauer effect studies carried out on a wide variety of mixed valence manganites as well as other types of magnetoresistive materials, including pyrochlore $\mathrm{Tl}_{2} \mathrm{Mn}_{2} \mathrm{O}_{7}$ and the chalcospinels $\mathrm{Fe}_{0.5} \mathrm{Cu}_{0.5} \mathrm{Cr}_{2} \mathrm{~S}_{4}$ and $\mathrm{FeCr}_{2} \mathrm{~S}_{4}$ with absolutely different nature of magnetism, in a search for similarities linked to their magnetoresistive behavior. Double exchange electron transfer and coupling between the electrons and Jahn-Teller lattice distortions invoked by most theories to explain colossal magnetoresistivity and associated metal-insulator transition in manganites are not applicable neither to pyrochlore nor to chalcospinels. Nevertheless, we find intriguing similarities in anomalous magnetic and dynamic behavior among these widely different systems at, above and below the Curie temperature $T_{C}$, which shed light on the origin of bulk magnetoresistivity in general. All these compounds share the following features. The long range ferromagnetic order breaks down even below the Curie temperature with the formation of nano size spin clusters. Softening of lattice was observed near $T_{C}$. The short range interactions in these spin clusters survive well above $T_{C}$. When an external magnetic field is applied, the spin clusters coalesce to form large clusters with considerable lowering of resistivity. There is a strong evidence that the existence of nano size spin clusters with superparamagnetic-like behavior near $T_{C}$ is a prerequisite to occurrence of bulk magnetoresistity.
\end{abstract}

PACS: 76.80.+y

In honor of V.V. Eremenko

\section{Introduction}

The perovskite manganite, $\mathrm{LaMnO}_{3}$ is an insulating antiferromagnet. The $\mathrm{Mn}-\mathrm{O}$ octahedra are highly distorted due to Jahn-Teller effect. When $\mathrm{La}^{3+}$ is substituted by bivalent ion like $\mathrm{Ca}^{2+}$, a corresponding amount of $\mathrm{Mn}^{3+}$ is converted to $\mathrm{Mn}^{4+}$. For instance, with the introduction of holes, the compound $\mathrm{La}_{1-x} \mathrm{Ca}_{x} \mathrm{MnO}_{3}$ becomes a ferromagnetic metal for $0.18<x<0.5$. The hopping of an electron from $\mathrm{Mn}^{3+}$ to $\mathrm{O}^{2-}$ is accompanied by a simultaneous hop from the latter to $\mathrm{Mn}^{4+}$. The probability of this double exchange (DE) electron transfer of an $e_{g}$ electron depends on the orientation of the neighboring intra-atomic Hund's coupled $t_{2 g}$ spins. The hopping is facilitated if the spins are aligned parallel to each other. Thus the ferromagnetic alignment of spins and the rate of electron hopping which is responsible for its metallicity are synergistically tied to each other [1]. The probability of the hopping also depends on the $\mathrm{Mn}^{3+}-\mathrm{O}-\mathrm{Mn}^{4+}$ bond angle. The latter is determined by the size of the rare earth/bivalent substituent ions. Among the four $3 d$ electrons of the $\mathrm{Mn}^{3+}$, the $t_{2 g}^{3}$ electrons are weakly hybridized and constitute the localized spin $(S=3 / 2)$. In contrast, $e_{g}^{1-x}$ orbitals have lobes directed to neighboring oxygen atoms, hybridize strongly with $\mathrm{O}_{2 p}$ orbitals and the electron is itinerant below $T_{C}$ in the ferromagnetic-metallic state and is believed to be localized in the «paramagnetic»-insulating state above $T_{C}$. The resistivity increases considerably as one approaches $T_{C}$ from below. On application of a magnetic field, there is a sharp decrease in resistivity due to regeneration of metallicity/ferromagnetism. This effect is known as «colossal magnetoresistivity (CMR)». To ex- 
plain this surprising behavior, several researchers have invoked the concept that the mobile electron carries with it the Jahn-Teller ( J-T) distortion of the $\mathrm{MnO}_{6}$ octahedron. The greater the distortion, the more localized are the charge carriers; this deformation disappears in the metallic state below $T_{C}$ [2-5]. In the ferromagnetic metallic state, there is a strong exchange interaction between the itinerant $\mathrm{e}_{g}$ electrons and the localized $\mathrm{t}_{2 g}$ spins. The basic problems facing the researchers are the understanding of features driving the ferromagnetic metal to «paramagnetic» insulator transition and the intriguing observation of large CMR upon application of modest external magnetic fields.

There are other systems, for instance, the spinel chalcogenide $\mathrm{FeCr}_{2} \mathrm{~S}_{4}$ [6-12], and the pyrochlore $\mathrm{Tl}_{2} \mathrm{Mn}_{2} \mathrm{O}_{7}$ [13-16] which exhibit CMR behavior. However, these systems do not show DE electron transfer nor Jahn-Teller distortions. In this paper, we will compare the magnetic and dynamic behavior of different families of CMR compounds and seek commonalities which could lead to a better understanding of the origin of bulk magnetoresistivity.

For our studies, we use a local probe, namely ${ }^{57}$ Co. Emission Mössbauer spectroscopy constitutes a valuable tool for exploring the behavior of materials at the microscopic level. It is couple of orders of magnitude more sensitive than the regular absorption mode and requires only a few tens of parts-per-million of $\mathrm{Mn}$ substitution by ${ }^{57} \mathrm{Co}$ with minimal perturbation of the system. On the other hand, one has to use $1-2 \%{ }^{57} \mathrm{Fe}$ substitution for the regular absorption mode. The minuscule amount of the ${ }^{57} \mathrm{Co}$ substituent faithfully probes the subtle changes in electron density, local dynamics, lattice distortions, and magnetic behavior of the dominant host material. The probe can also sense the formation of different electronic phases. We have shown earlier [17-19] that the Auger after-effects do not cause any problem in systems having delocalized electrons since the 50-100 eV excitation energy deposited at the epicenter/molecule (incorporating ${ }^{57} \mathrm{Co}$ ) during electronic relaxation of the multiply charged site goes towards collective excitation of delocalized electrons. The plasmon decays in less than $10^{-14} \mathrm{~s}$ transferring practically all its energy to an ejected electron. The strong coupling between plasmons formed from delocalized electrons and single electron continuum states provides a neat mode of energy disposal leaving the ${ }^{57} \mathrm{Fe}$ site virtually unperturbed [17-19]. The $14.4 \mathrm{keV}$ Mössbauer gamma rays which are dressed with information about the chemical environment are emitted with $\sim 10^{-7}$ s delay after the electron-capture nuclear decay and are therefore oblivious of the Auger event. The formation of a plasmon by excitation of delocalized electrons by synchrotron radiation and its decay by ejection of an electron has been verified by photoemission studies of $\mathrm{C}_{60}$ and $\mathrm{C}_{70}[20,21]$. Later, we will make use of this feature of emission Mössbauer spectroscopy to buttress our claim that the CMR materials are insulating above $T_{C}$ in the macroscopic sense only. On the microscopic scale, small magnetic clusters are formed with delocalized electrons. The delocalized electrons protect the site from suffering local radiation damage following Auger ionization.

\section{CMR manganites}

\section{Superparamagnetic-like behavior below and above $T_{C}$}

Typical Mössbauer spectra of manganites as a function of temperature is represented by $\mathrm{La}_{0.8} \mathrm{Ca}_{0.2} \mathrm{Mn}\left({ }^{57} \mathrm{Co}\right) \mathrm{O}_{3}$ in Fig. 1,a [22]. Spectra showing similar behavior were reported earlier also [23-27]. The anomalous magnetic behavior of mixed valence CMR manganites becomes obvious
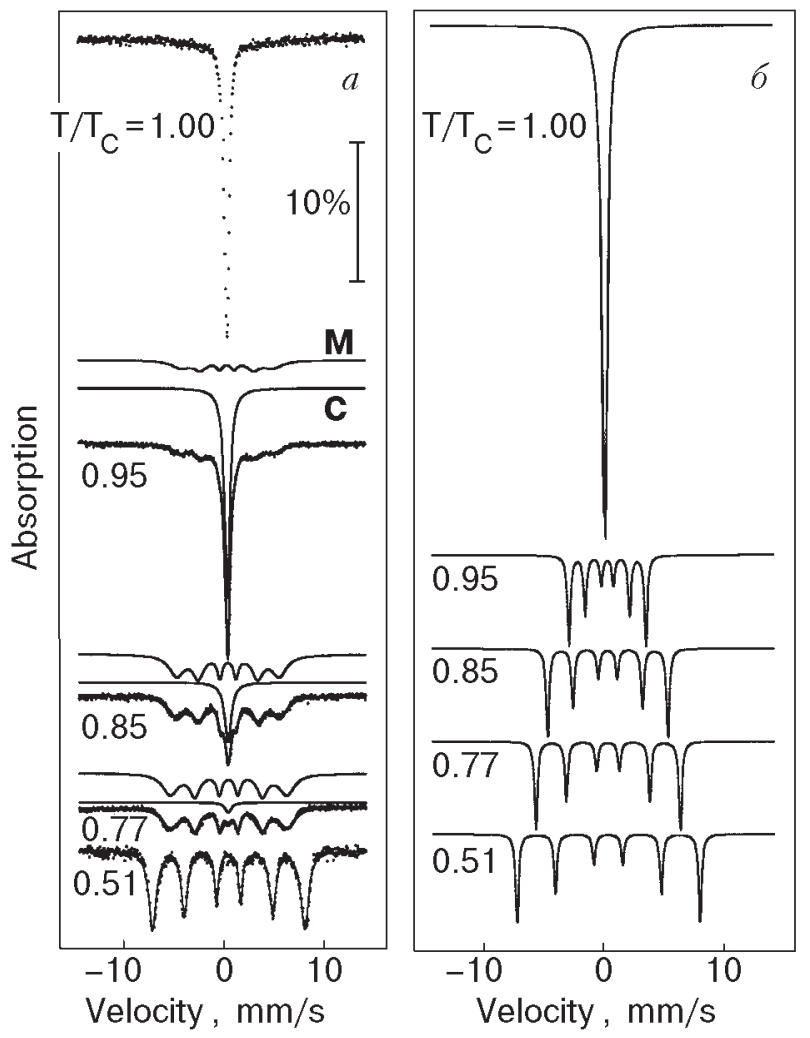

Fig. 1. Mössbauer spectra of $\mathrm{La}_{0.8} \mathrm{Ca}_{0.2} \mathrm{Mn}\left({ }^{57} \mathrm{Co}\right) \mathrm{O}_{3}$ at different temperatures, $T_{C}=198 \mathrm{~K}(a)$; computer simulated Mössbauer spectra of a regular ferromagnet at various temperatures (b) [Ref. 22]. 
by comparison with the computer simulated spectra for a regular ferromagnet given in Fig. 1,b. In a regular ferromagnet, the spectral extent of the magnetically split sextet which is proportional to the natural magnetization, diminishes continuously with temperature and finally collapses into a single line at $T_{C}$. On the other hand, for the manganite, the most important difference consists in observation of two components as one approaches $T_{C}$ from
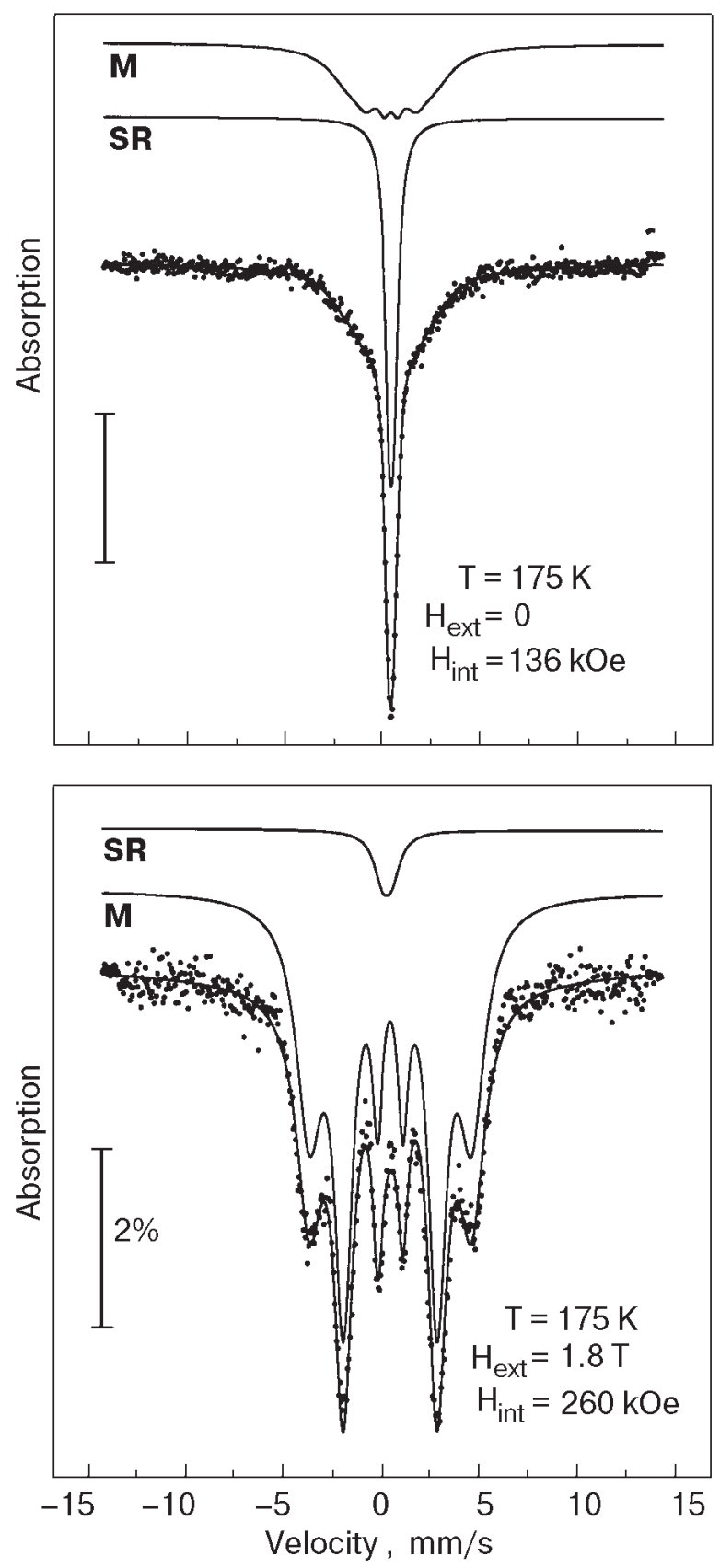

Fig. 2. Mössbauer spectra of the $\mathrm{La}_{0.9} \mathrm{Ca}_{0.1} \mathrm{Mn}\left({ }^{57} \mathrm{Co}\right) \mathrm{O}_{3}$ single crystal with and without $H_{\text {ext }}=1.8 \mathrm{~T}$ at $T=$ $=175 \mathrm{~K}\left(T / T_{C}=0.99\right)$. The computer fits are raised for clarity [Ref. 28]. below, a sextet $M$ and a central peak $C$. In a regular ferromagnet we would expect to see the $C$ component only above $T_{C}$.

In manganites, the coexisting central component grows at the expense of the sextet with increasing temperature. Such behavior is typical for superparamagnetic behavior of nano size single domain particles with a distribution in size. The grain size in all our polycrystalline samples is larger that $1 \mu \mathrm{m}$. Moreover, a single crystal of $\mathrm{La}_{0.9} \mathrm{Ca}_{0.1} \mathrm{Mn}\left({ }^{57} \mathrm{Co}\right) \mathrm{O}_{3}$ also shows similar behavior [28], and so we conclude that it is an intrinsic property of manganites that the long range ferromagnetic order breaks down even below $T_{C}$ and nano size magnetic clusters are formed with shortrange order. If the available thermal energy at any temperature is sufficient to overcome the anisotropy barrier, then the magnetic vector of the spin clusters can flip among the easy directions at a rate faster than the Larmor frequency of the daughter ${ }^{57} \mathrm{Fe}$ (about $10^{8} \mathrm{~s}^{-1}$ ), then the internal magnetic field at the probe averages out to zero. Manganites exhibit another very interesting feature which contrasts with regular superparamagnetic behavior. The blocking temperature (the temperature at which the sextet collapses completely into a singlet/doublet when the rate of fluctuations of the magnetic vectors of all the clusters exceeds the Larmor frequency) coincides with the Curie temperature. This means that as we approach $T_{C}$ from below, an increasing number of nano spin clusters are

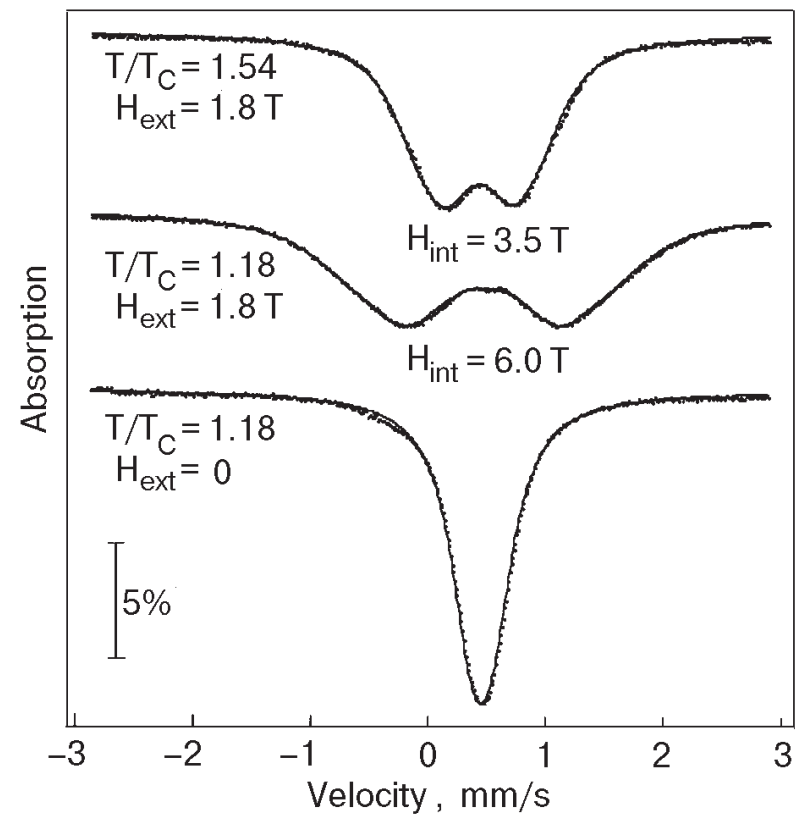

Fig. 3. Effect of $H_{\text {ext }}=1.8 \mathrm{~T}$ on the Mössbauer spectra of $\mathrm{La}_{0.8} \mathrm{Ca}_{0.2} \mathrm{Mn}\left({ }^{57} \mathrm{Co}\right) \mathrm{O}_{3}$ at $T / T_{C}=1.18$ and 1.54 , $T_{C}=198 \mathrm{~K}$ [Ref. 26] 
formed from the bulk, there is always some fraction of the material exhibiting bulk-like magnetism even up to $0.99 T_{C}$ (Fig. 2) $[28,29]$. The sextet lines are rather broad even at $T / T_{C} \sim 0.3$ and become broader with increasing temperature (Fig. 1,a). Only at very low temperatures $\left(\mathrm{T} / T_{C} \sim 0.05\right)$ the sextet looks like that of a regular ferromagnet (Fig. 1,b). This broadening occurs partly due to collective excitations of clusters in directions close to the easy ones. Moreover, exchange interactions also depend on the degree of spin alignment which varies with the cluster size resulting in a distribution of internal hyperfine magnetic fields experienced by the Mössbauer probe which tend to increase with temperature.

\section{Influence of external magnetic field}

The question arises whether the collapsed singlet both below $T_{C}$ as well as above is really due to «superparamagnetically relaxed» spin clusters or due to ordinary paramagnetic species. We can get the answer by observing the Mössbauer spectrum near $T_{C}$ in a modest external magnetic field ( $H_{\text {ext }}$. One can see from Fig. 2 a dramatic change in the Mössbauer spectrum with the application of $H_{\text {ext }}=$ $=1.8 \mathrm{~T}$. One observes a regular magnetically split spectrum characteristic of bulk ferromagnet with induced internal magnetic field $H_{\text {int }}=260 \mathrm{kOe}$. This is a very graphic evidence of coalescence of pre-existing nano size clusters to form large clusters with well ordered ferromagnetic alignment.

The next question is whether these nano spin clusters survive well above $T_{C}$. If they are not, one will not be able to rationalize the observation of magnetoresistivity above the Curie temperature. Figure 3 depicts changes in the Mössbauer spectrum at $T=1.18 T_{C}$ and $T=1.54 T_{C}$ in $H_{\text {ext }}=1.8 \mathrm{~T}$. The spectra can be fit by a magnetically split sextet with $H_{\text {int }}=60$ and $35 \mathrm{kOe}$, respectively, and the line intensity ratio $3: 4: 1: 1: 4: 3$ corresponding to the sample magnetization perpendicular to the gammaray direction. These observations clearly indicate the presence of pre-existing nano spin clusters with each one having adequate total spin to react to a very modest $H_{\text {ext }}$ at temperatures comparable to room temperature, and consolidate into larger clusters as indicated by relatively robust $H_{\text {int }}$ (Fig. 3). The interaction between spin clusters aided by $H_{\text {ext }}$ is synergetic, i.e., the better alignment of spins results in improving DE electron transfer/metallicity which in turn enhances spin ordering. Therefore, a direct correlation exists between the change in the abundance of the ferromagnetic fraction and the magnetoresistivity as a function of temperature on application of $H_{\text {ext }}$ [26]. Also a linear dependence of the resistance on the magnitude of ferromagnetic fraction in zero $H_{\text {ext }}$ in $\mathrm{La}_{0.8} \mathrm{Ca}_{0.2} \mathrm{Mn}\left({ }^{57} \mathrm{Co}\right) \mathrm{O}_{3}$ was observed [25]. These observations are based on simultaneous measurements of resistance and emission Mössbauer spectra. The observed correlation suggests that the current flows almost exclusively through filamentary ferromagnetic regions surrounded by superparamagnetic nano spin clusters [25,30,31]. It may be mentioned that the above discussed evidence for existence of nano spin clusters (magnetic polarons) above $T_{C}$ can be further reinforced by the following technique. If spin clusters really exist above $T_{C}$, there is a probability that some of them could be relatively large and fluctuate slowly enough with relaxation time in a range centered at about $10^{-9} \mathrm{~s}$. The Mössbauer spectra originating from such clusters may consist of a semicollapsed central part with very wide wings [32,33]. It is easy to lose these extensive wings in the background during computer analysis, while the central part can incorrectly be analyzed to be a part of the completely superparamagnetically relaxed component originating from the major fraction of smaller spin clusters. If the energy scale during the measurements is deliberately adjusted to cut the extensive wings and accommodate only the collapsed central component, it becomes possible to determine indirectly the existence of such type of clusters above $T_{C}$ by observing the normalized area of the spectrum as a function of temperature. Experiment shows that as the temperature is lowered toward $T_{C}$, the abundance (normalized area) instead of increasing, as expected according to the theory, goes down - the closer to the $T_{C}$, more is the observed loss. This suggests that by approaching $T_{C}$ from above and keeping a narrow energy window, we lose gradually increasing amounts of spectral area associated with the fraction of clusters whose fluctuating rate becomes less than about $10^{9} \mathrm{~s}^{-1}$ [29].

De Teresa and coworkers [34] made volume thermal expansion with and without $H_{\text {ext }}$, magnetic susceptibility, and small angle neutron scattering (SANS) measurements. They interpreted their observations by invoking presence of magnetic polarons dispersed in a paramagnetic matrix. Our observations given in Figs. 2 and 3 do not bear out the presence of paramagnetic matrix: all the nano spin clusters present are equally affected and grow under the influence of $H_{\text {ext }}$. It may be mentioned that a specific spin does not belong always to the same spin cluster but there is a continuous exchange of spins between the clusters. The dynamics 


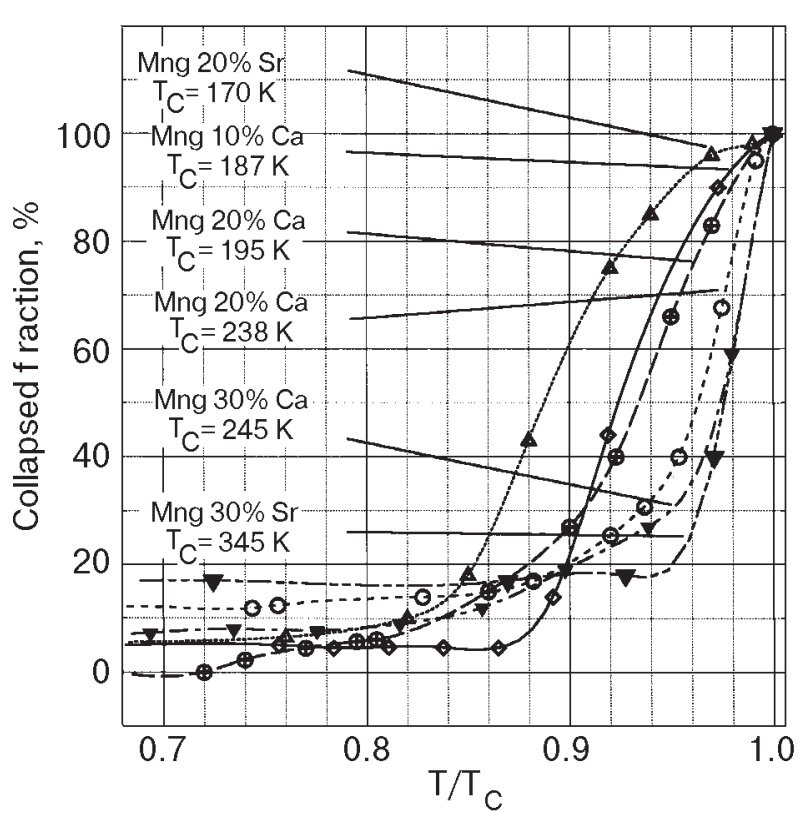

Fig. 4. Plot of superparamagnetically collapsed fraction vs $T / T_{C}$ for variety of CMR manganites obtained by computer analysis of the temperature evolution of their emission Mössbauer spectra. Different $T_{C}$ 's for the samples with the same degree of substitution is a result of different oxygen content.

of the exchange has to be slower than the Larmor period of ${ }^{57 \mathrm{~m}} \mathrm{Fe}\left(\sim 10^{-8} \mathrm{~s}\right)$, otherwise the probe nuclei would not sense the internal hyperfine field and instead of the sextet one would observe a completely collapsed spectrum [22,35]. Related to it are the observations made by Raquet et al. [36] in $\mathrm{La}_{2 / 3} \mathrm{Ca}_{1 / 3} \mathrm{MnO}_{3}$ films of giant random telegraph noise in the resistance fluctuations well below $T_{C}$. They attribute it to the dynamic mixed-phase percolative conduction process, where manganese clusters switch back and forth between two phases that differ in their conductivity and magnetization. Similar observations were made by Podzorov et al. [37] and Zhang et al. [38]. In this compound, apart from two ferromagnetic phases $[39,40]$, we also detect nano size magnetic clusters well below $T_{C}$ [39] which can help to understand the observations made by the above mentioned researches.

Taking into consideration the superparamagnetic-like behavior of mixed valence manganites at different temperatures [22-29,39] and the interaction energy between a modest $H_{\text {ext }}$ and the nano spin clusters (Fig. 3), we estimate approximately the average size of the magnetic clusters as roughly $20 \AA$ in diameter (if approximated by a sphere) containing couple of hundred $\mathrm{Mn}^{3+} / \mathrm{Mn}^{4+}$ atoms. The distribution of cluster size and the temperature

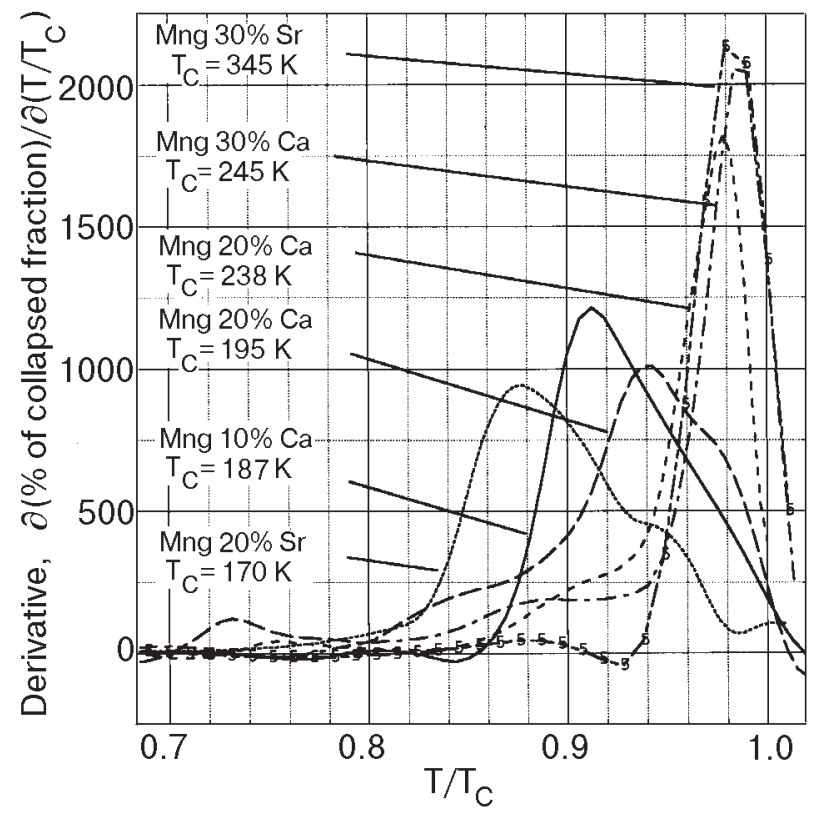

Fig. 5. Derivative of the results presented in Fig. 4 which reflects the rate of cluster formation from the bulk with temperature as well as the width of cluster size distribution.

range in which they are formed from the bulk near $T_{C}$ varies with the fraction of bivalent ion $\left(\mathrm{Ca}^{2+}\right.$, $\mathrm{Sr}^{2+}$ ) substitution for $\mathrm{La}^{3+}$. For $30 \% \mathrm{Ca} / \mathrm{Sr}$ substituted compound it was found to be narrower than for compounds with 10 and $20 \%$ Ca substitution as discussed below. Figure 4 shows the supermagnetically relaxed fraction as a function of $T / T_{C}$. The derivative of this plot gives a qualitative feel for the distribution of cluster size (Fig. 5). However, we have to keep in mind that at any given temperature, we are not dealing with pre-existing magnetic clusters with a large distribution in size, but rather a narrow enough distribution with increasing number of clusters being formed from the bulk as the temperature is increased. As seen from Fig. 4, there is a definite correlation between magnetic quality of the compound (represented by $\mathrm{Cu}-$ rie temperature) and the temperature range where transformation takes place: the higher the $T_{C}$ the narrower the range. It seems that the better the ferromagnetic material (with higher conductivity) it will have more homogeneous clusters with a narrower distribution in size. At the same time, it is known that CMR effect decreases with the increase of $T_{C}$. We also observe smaller effect of $H_{\text {ext }}$ on Mössbauer spectra of samples with higher Curie temperature. We attribute this to smaller average cluster size in compounds with higher $T_{C}$. 


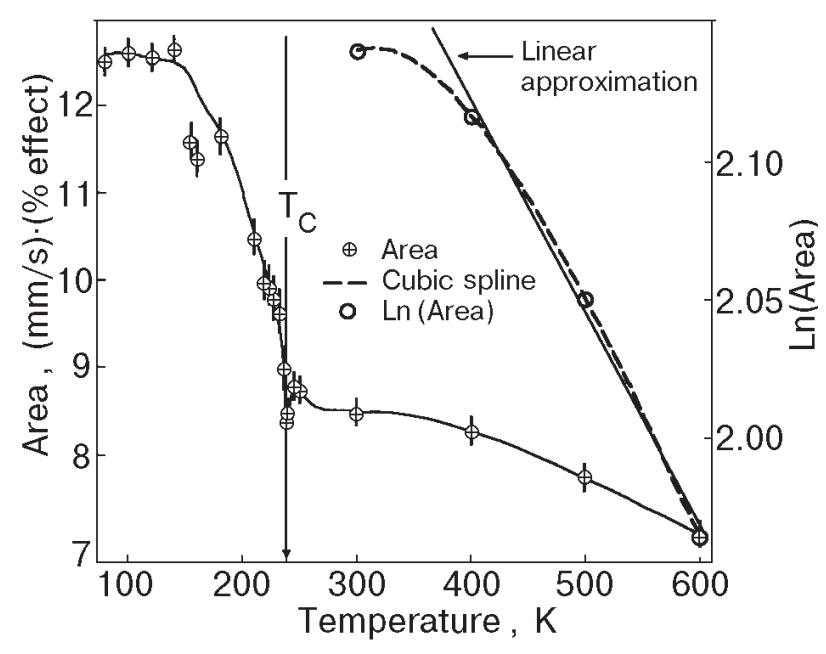

Fig. 6. Plot of normalized total area under the Mössbauer spectra of $\mathrm{La}_{0.8} \mathrm{Ca}_{0.2} \mathrm{Mn}\left({ }^{57} \mathrm{Co}\right) \mathrm{O}_{3}$ as a function of temperature. The solid line is a guide for the eyes.

\section{Model}

Now we have to address the question as to how the long range ferromagnetic order breaks down with formation of nano size magnetic clusters below $T_{C}$ and the survival of the short range order in the spin clusters up to a temperature close to $2 T_{C}$ [26,29,30,35]. This behavior contrasts sharply with that of regular ferromagnets like $\mathrm{Fe}$ and $\mathrm{Ni}$, where magnetic correlations are observed only up to $1.1 T_{C}$, and there is no evidence of formation of spin clusters below $T_{C}$. A very revealing observation made by Mössbauer studies was the sharp drop in the total Mössbauer spectral area when approaching $T_{C}$ from below [25,35]. The magnitude of the Mössbauer area is indicative of how strongly the Mössbauer probe is bound to the $\mathrm{Mn}-\mathrm{O}-\mathrm{Mn}$ network. A sharp decrease below $T_{C}$ strongly suggests softening of the phonon spectra (Fig. 6). This softening occurs presumably due to the anharmonic stretching of $\mathrm{Mn}-\mathrm{O}$ bonds and the enhanced amplitude of torsional oscillations of the $\mathrm{Mn}-\mathrm{O}$ octahedra. The anomalous increase in $\mathrm{Mn}-\mathrm{O}$ bond lengths, bending of the $\mathrm{Mn}-\mathrm{O}-\mathrm{Mn}$ moiety and the enhanced Debye-Waller factors for $\mathrm{Mn}$ and $\mathrm{O}$ atoms below $T_{C}$ have also been observed by x-ray and neutron diffraction studies [41-47]. These observations have also been attributed to anharmonic oscillations by Dai et al. [41] and Rodrigues-Martinex and Attfield [45]. The anomalous increase in thermal expansion near $T_{C}$ can also be attributed to anharmonicity $[34,41,48,49]$. The localized distor- tions resulting from aliovalent substitution e.g. $\mathrm{Ca}^{2+}$ and $\mathrm{Sr}^{2+}$ are presumably responsible for the anomalous anharmonicity. The stretching of bonds and the larger amplitude of torsional oscillations between $\mathrm{Mn}-\mathrm{O}$ octahedra would decrease the exchange interactions and also the rate of DE electron hopping, the two parameters are intertwined synergistically. For instance, if the amplitude of the torsional oscillations increase with temperature, the rate of electron hopping will decrease as the neighboring spins will have to wait until thermal fluctuations allow them an appropriate configuration for a double exchange hop (Franck-Condon factor). Therefore, with increasing temperature below $T_{C}$, the long range ferromagnetic order breaks down and nano size ferromagnetic clusters with short range interactions and superparamagnetic-like fluctuations appear. The fluctuations are responsible for strong electron scattering. On application of an external magnetic field, nano spin clusters (which survive even well above $T_{C}$ ) tend to coalesce and grow in size. If the clusters become large enough to exhibit conductivity by percolation, CMR will be observed [24-26,30,31]. One of the parameters determining the magnitude of CMR depends on the degree of alignment of spin clusters upon application of $H_{\text {ext }}$ which in turn depends on the initial cluster size. Our work seems to suggest that the cluster size increases with decrease in $\mathrm{Cu}^{-}$ rie temperature of the material.

Now the question arises whether these magnetic (clusters)polarons are dressed with lattice distortions. The Mössbauer spectra above $T_{C}$ for all studied manganites show very small quadrupole splitting (about $0.18 \mathrm{~mm} / \mathrm{s}$ ) [22,29] (and becomes even smaller below $T_{C}$ ) which indicates that the distortions imposed on the octahedron incorporating the Mössbauer probe by the surrounding $\mathrm{Mn}-\mathrm{O}$ network is quite small. The probe senses distortions in a $10^{-7} \mathrm{~s}$ period. Our observation of a single major species above $T_{C}$ is supported by ESR studies on $\mathrm{La}_{1-x} \mathrm{Sr}_{x} \mathrm{MnO}_{3}(x=0.1,0.2$, and 0.3$)$ by Lofland et al. [50]. They find that all $\mathrm{Mn}$ spins contribute to the spectrum above $T_{C}$ indicating formation of magnetic clusters. Similarly, Kapusta et al. [51] report the observation of a single ${ }^{55} \mathrm{Mn}$ NMR line both below and above Curie temperature for $\mathrm{La}_{1-x} \mathrm{~A}_{x} \mathrm{MnO}_{3}(\mathrm{~A}=\mathrm{Ca}, \mathrm{Sr})$ providing direct evidence of magnetic correlations on a time scale greater than $10^{-5} \mathrm{~s}$. These results obtained by techniques which operate on relatively slow time scale are in apparent contradiction with observations made by much faster methods. The x-ray absorbtion fine structure spectroscopy and pair distribution 
function studies on CMR manganites show lattice distortions above $T_{C}$ [2-5]. Neutron scattering investigations also give evidence of distortion in the insulating state above $T_{C}$ [41-47]. Moreover, the spin stiffness coefficient does not go to zero at $T_{C}$ which indicates existence of short range magnetic interactions as in magnetic clusters (polarons). Two recent reports which bear directly on our model are worthy of mention. Dai et al. [52] and Adams et al. [53] using neutron scattering have demonstrated the presence of polarons even below $T_{C}$ in $\mathrm{La}_{1-x} \mathrm{Ca}_{x} \mathrm{MnO}_{3}(x=0.2$ and 0.3$)$. They also observe short range polaron correlations with a coherence range of $\sim 28 \AA$ just above Curie temperature. Here the time scale of observations is several order of magnitude shorter than that for Mössbauer method. We feel that their results are consistent with our model; they are observing magnetic clusters but with larger distortions than us just because the distortions detected in time scale of $\sim 10^{-12} \mathrm{~s}$ would be averaged out in the Mössbauer time scale of $\sim 10^{-7} \mathrm{~s}$, if they are of a dynamic nature. The frequency of Jahn-Teller oscillations is reduced near $T_{C}$ due to slowing down of DE electron transfer

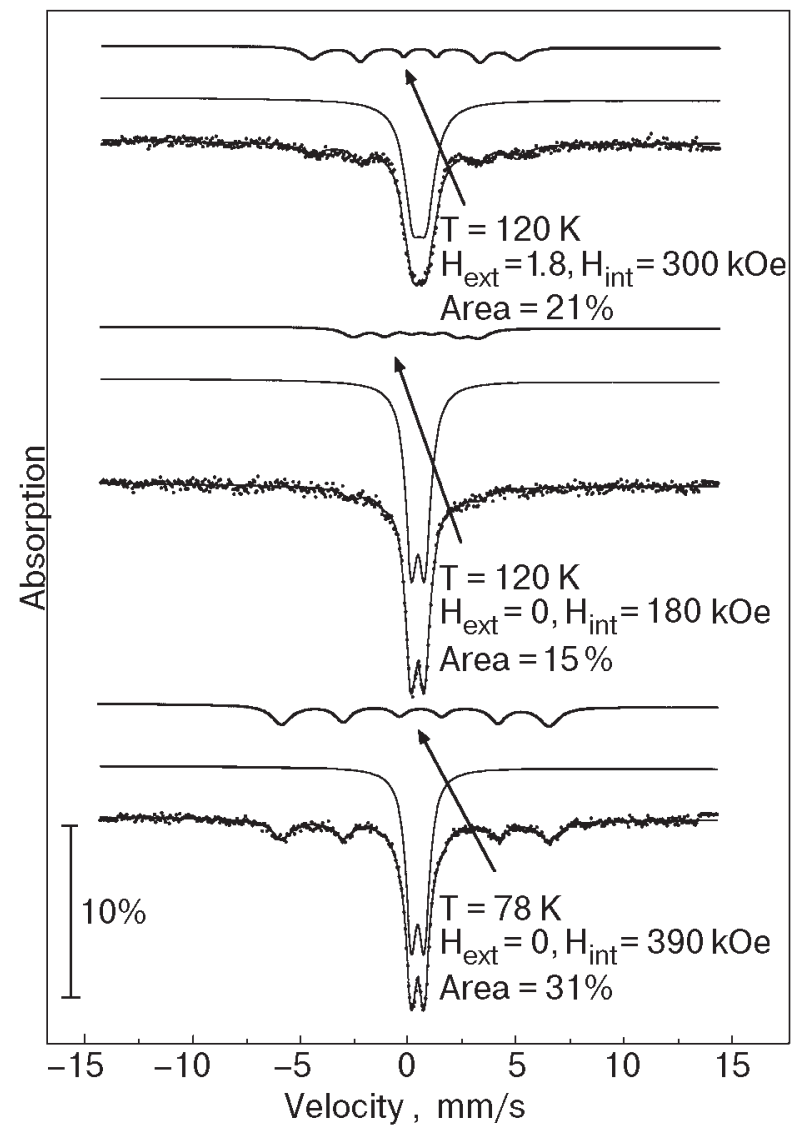

Fig. 7. Mössbauer spectra of the pyrochlore, $\mathrm{Tl}_{2} \mathrm{Mn}_{2}\left({ }^{57} \mathrm{Co}\right) \mathrm{O}_{7}\left(T_{C}=123 \mathrm{~K}\right)[22]$. and so neutron scattering can detect the distortions. However, they are still fast for the Mössbauer time window.

\section{Other CMR Compounds}

Recently, there have been reports of some non manganite-based systems which also exhibit magnetoresistivity. Among them, the pyrochlore $\mathrm{Tl}_{2} \mathrm{Mn}_{2} \mathrm{O}_{7}$ and the chalcospinels $\mathrm{Fe}_{0.5} \mathrm{Cu}_{0.5} \mathrm{Cr}_{2} \mathrm{~S}_{4}$ and $\mathrm{FeCr}_{2} \mathrm{~S}_{4}$, where neither $\mathrm{DE}$ transfer between aliovalent ions nor Jahn-Teller distortions occur. The pyrochlore, $\mathrm{Tl}_{2} \mathrm{Mn}_{2} \mathrm{O}_{7}$. shows $\mathrm{CMR}$ without $\mathrm{Mn}^{3+} / \mathrm{Mn}^{4+} \mathrm{DE}$. Its formal oxidation states as detected by x-ray diffraction are $\mathrm{Tl}_{2}{ }^{3+} \mathrm{Mn}_{2}{ }^{4+} \mathrm{O}_{7}$ $[13,14]$. Calculations show that the Fermi surface is derived from strongly mixed combinations of $\mathrm{Tl}$, $\mathrm{Mn}$, and O orbitals. $[15,16]$. Our preliminary emission Mössbauer investigations show evidence of the existence of nano size ferromagnetic clusters in this compound at and below the Curie temperature $T_{C}=$ 123K [22,54]. The Mössbauer spectra of $\mathrm{Tl}_{2} \mathrm{Mn}_{2}\left({ }^{57} \mathrm{Co}\right) \mathrm{O}_{7}$ at 78 and $120 \mathrm{~K}$ without external magnetic field and at $120 \mathrm{~K}$ with $H_{\text {ext }}=1.8 \mathrm{~T}$ are shown in Fig. 7. One can see a coexistence of collapsed and magnetically split components. When the temperature is raised from $78 \mathrm{~K}$ to $120 \mathrm{~K}$, the area of the sextet decreases from $31 \%$ to $15 \%$ with the corresponding increase of the area of collapsed component, and the internal hyperfine magnetic field decreases from $390 \mathrm{kOe}$ to $180 \mathrm{kOe}$. Upon application of $H_{\text {ext }}=1.8 \mathrm{~T}$ at $T=120 \mathrm{~K}$, not only the area of the sextet increases to $21 \%$ but most importantly $H_{\text {int }}$ increases to $300 \mathrm{kOe}$. We expect that lowering the temperature to $T / T_{C} \sim 0.15$ would result in a spectrum consisting of mainly magnetically ordered component. In short, the changes observed in Mössbauer spectra of $\mathrm{Tl}_{2} \mathrm{Mn}_{2}\left({ }^{57} \mathrm{Co}\right) \mathrm{O}_{7}$ as a function of temperature and under influence of $H_{\text {ext }}$ are similar to that of the manganites discussed earlier. It allows us to assign with confidence the superparamagnetic nature to the central collapsed component originating from the existence of nano size magnetic clusters.

Lang et al. [55] have reported ${ }^{57} \mathrm{Fe}$ Mössbauer spectra of the CMR chalcospinel $\mathrm{Fe}_{0.5} \mathrm{Cu}_{0.5} \mathrm{Cr}_{2} \mathrm{~S}_{4}$ at temperatures ranging from $4 \mathrm{~K}$ to $300 \mathrm{~K}$. From their figures one can observe the coexistence of a central peak and a sextet at all temperatures below $300 \mathrm{~K}$. The central peak increases in intensity at the expense of the sextet as the temperature is increased. The authors do not discuss this behavior. However, we would like to attribute it to superparamagnetic-like behavior of spin clusters 


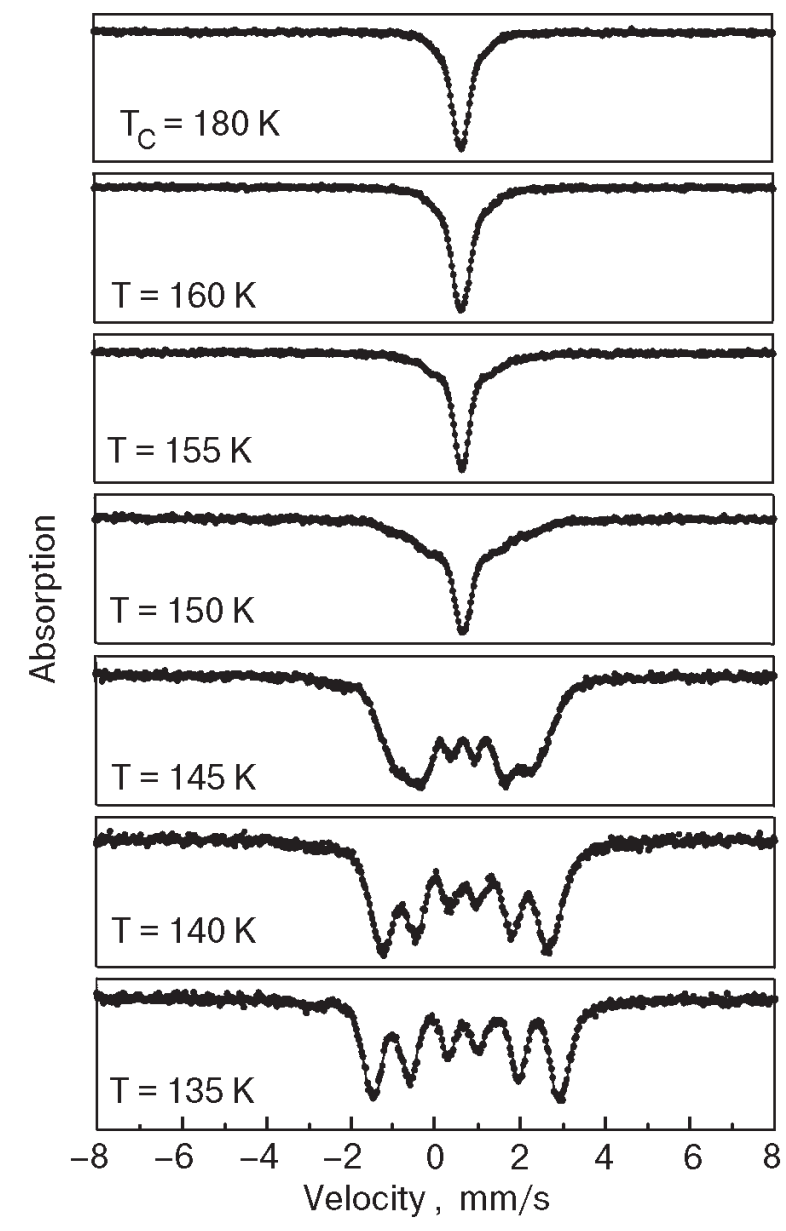

Fig. ${ }^{8} .{ }^{57} \mathrm{Fe}$ Mössbauer spectra of $\mathrm{FeCr}_{2} \mathrm{~S}_{4}$ taken at selected temperatures at and below the magnetic ordering temperature $\left(T_{C}=180 \mathrm{~K}\right)$ [Ref. 56].

which form in increasing amounts from the bulk as one approaches $T_{C}$ from below.

We have recently studied another chalcospinel $\mathrm{FeCr}_{2} \mathrm{~S}_{4}$ using ${ }^{57} \mathrm{Fe}$ absorption method [56] in the temperature range $78-300 \mathrm{~K}\left(T_{C}=180 \mathrm{~K}\right) . \mathrm{FeCr}_{2} \mathrm{~S}_{4}$ is a $p$-type semiconducting ferrimagnet with the Curie temperature above $175 \mathrm{~K}$. The formal charges are $\mathrm{Fe}^{2+}, \mathrm{Cr}^{3+}$, and $\mathrm{S}^{2-}$ with considerable covalent character. $\mathrm{Fe}^{2+}$ occupy tetrahedral sites and $\mathrm{Cr}^{3+}$ octahedral ones. They are aligned antiferromagnetically. Double exchange electron transfer in this compound is ruled out. Some representative experimental data are shown in Fig. 8. Features previously discussed can be clearly seen from this figure. At low temperature one observes only one component - a well defined sextet. As the temperature increases, a central collapsed component emerges gradually growing at the expense of the sextet. We also found that the total area of the spectrum shows a sharp decrease between 145 and $170 \mathrm{~K}$ indicating softening of the lattice, a feature similar to that observed by us for all CMR manganites. At a temper- ature of $186 \mathrm{~K}$, i.e., above $T_{C}$, application of an external magnetic field of $6 \mathrm{~T}$ restores well defined sextet typical for bulk the ferromagnet indicating the coalescence of nano spin clusters.

On the basis the these observations one can infer that in many respects CMR pyrochlore and chalcospinels demonstrate similar behavior, namely: (i) the long range ferromagnetic order breaks down gradually when approaching $T_{C}$ from below, with formation of spin clusters; (ii) nano size spin clusters survive well above $T_{C}$; (iii) application of an external magnetic field at, as well as above $T_{C}$ causes coalescence of those clusters with restoration of bulk-like ferromagnetism, and lowering of resistivity. These common features among widely different magnetoresistive materials indicate that existence of nano size clusters with superparamagnetic-like behavior is a prerequisite to observing bulk negative magnetoresistivity in such systems.

\section{Acknowledgments}

AN thanks the donors of the PRF administered by ACS for partial support of this research.

1. C. Zener, Phys. Rev. 82, 403 (1951); P. W. Anderson and H. Hasegawa, Phys. Rev. 100, 675 (1953).

2. J. M. D. Coey, M.Viret, and S. von Molnar, Adv. Phys. 48, 167 (1999).

3. Colossal Magnetoresistive Oxides, Y. Tokura (ed.), Gordon and Breach Sci. Publ. Amsterdam (2000).

4. E. L. Nagaev, Phys. Rep. 346, 387 (2001).

5. M. B. Salomon and M. Jaime, Rev. Mod. Phys. 73, 583 (2001).

6. P. F. Bongers, C. Haas, A. M. J. G. van Run, and G. Zanmarchi, J. Appl. Phys. 40, 958 (1969).

7. T. Watanabe, Solid State Commun. 12, 355 (1973).

8. K. Ando, Y. Nishihara, T. Okuda, and T. Tsushima, J. Appl. Phys. 50,1917 (1979).

9. A. P. Ramirez, R. J. Cava, and J. Krajewski, Nature 386, 156 (1997).

10. Z. Chen, S. Tan, Z. Yang, and Y. Zhang, Phys. Rev. B59, 11172 (1999).

11. Z. Yang, S. Tan, Z. Chen, and Y. Zhang, Phys. Rev. B62,13872 (2000).

12 E. Z. Kurmaev, A. V. Postnikov et al., J. Phys. Condens. Matter 12, 5411 (2000).

13. M. A. Subramanian, B. H. Toby, A. P. Ramirez, W. J. Marshall, A. W. Sleight, and G.H. Kwei, Science 273, 81 (1996).

14. G. H. Kwei, C. H. Booth, F. Bridges, and M. A. Subramanian, Phys. Rev. B55, R688 (1997).

15. D. J. Singh, Phys. Rev. B55, 313 (1997).

16. Y. Shimakawa and Y. Kubo, Mater. Sci. Eng. B63, 44 (1999). 
17. M. B. Misrock, C. J. Schramm, and A. Nath, J. Chem. Phys. 65, 1982 (1976).

18. A. Nath, Ch. Sauer, and A. Halpern, J. Chem. Phys. 78, 5125 (1983).

19. A. Nath, M. Prushan, and J. G. Gilbert, J. Radioanalyt. Nucl. Chem. 247, 589 (2001).

20. I. V. Hertel, H. Steger, J. de Vries, B. Weisser, C. Menzel, B. Kamke, and W. Kamke, Phys. Rev. Lett. 68, 784 (1992).

21. S. Hunsche, T. Starczewski, A. l'Huillier, A. Persson, C.-G. Wahström, B. van den Heuvell, and S. Svanberg, Phys. Rev. Lett. 77, 1966 (1996).

22. A. Nath, V. Chechersky, and R. L. Greene, J. Solid State Chem. 55, 116 (2000). Invited contribution.

23. M. Pissas, G. Kallias, E. Devlin, A. Simopolous, and D. Niarchos, J. Appl. Phys. 81, 5770 (1997).

24. V. Chechersky, A. Nath, H.-L. Ju, and R. L. Greene, Fiz. Nizk. Temp. 23, 727 (1997) [Low Temp. Phys. 23, 545 (1997)].

25. V. Chechersky, K. Nomura, A. Nath, H.-L. Ju, and R. L. Greene, Fiz. Nizk. Temp. 23, 732 (1997) [Low. Temp. Phys. 23, 549 (1997)].

26. V. Chechersky, A. Nath, I. Isaac, J. P. Franck, K. Ghosh, H-L. Ju, and R. L. Greene, Phys. Rev. B59, 497 (1999).

27. V. Chechersky, A. Nath, I. Isaac, J. P. Franck, K. Ghosh, and R. L. Greene, Phys. Rev. B60, 3005 (1999)

28. V. Chechersky, A. Nath, S. E. Lofland, S. Newlander, L. Cerquoni, Y. Mukovskii, A. A. Arsenov, G. Karabshev, D. A. Shulyatev, and R. L. Greene, Phys. Rev. B63, 4401 (2001).

29. V. Chechersky, A. Nath, I. Isaac, J. P. Franck, K. Ghosh, and R. L. Greene, Phys. Rev. B63, 052411 (2001).

30. M. Jaime, P. Lin, S. H. Chun, M. B. Salamon, P. Dorsey, and M. Rubinstein, Phys. Rev. B60, 1028 (1999). S. H. Chun, M. B. Salamon, Y. Tomioka, and Y. Tokura, Phys. Rev. B61, R9225 (2000).

31. L. P. Gor'kov and V. Z. Kresin, JETP Letters 67, 985 (1998).

32. H. H. Wickmann, M. P. Klein, and D. A. Shirley, Phys. Rev. 152, 345 (1966).

33. S. Morup, Hyperfine Interact. 60, 959 (1990).

34. J. M. De Teresa, M. R. Ibarra, P. A. Algarabel, C. Ritter, C. Marquina, J. Blasco, J. Garcia, C. Fermon, A. del Moral, and Z. Arnold, Nature 386, 256 (1997).

35. V. Chechersky, A. Nath, I. Isaac, J. P. Franck, K. Ghosh, and R. L. Greene, J. Phys.: Condens. Matter 11, 8921 (1999).

36. B. Raquet, A. Anane, S. Wirt, P. Xiang, and S. von Molnar, Phys. Rev. Lett. 84, 4485 (2000).

37. V. Podzorov, M. E. Gershenson, M. Uehara, and S.-W. Cheong, Phys. Rev. D64, 115113 (2001).
38. J. Zhang, P. Dai, J. A. Fernandez-Baca, E. W. Plummer, Y. Tomioka, and Y. Tokura, Phys. Rev. Lett. 86, 3823 (2001).

39. V. Chechersky, A. Nath, C. Michel, M. Hervieu, K. Ghosh, and R. L. Greene, Phys. Rev. B62, 5316 (2000).

40. M. M. Savosta and P. Novak, Phys. Rev. Lett. 87, 137204 (2001).

41. P. Dai, J. Zhang, H. A. Mook, S.-H. Liou, P. A. Dowben, and E. W. Plummer, Phys. Rev. B54, R3694 (1996).

42. P. G. Radaelli, M. Marezio, H. Y. Hwang, S.-W. Cheong, and B. Batlogg, Phys. Rev. B54, 8992 (1996).

43. P. G. Radaelli, G. Iannone, M. Marezio, H. Y. Hwang, S.-W. Cheong, J. D. Jorgensen, and D. N. Argyriou, Phys. Rev. B56, 8265 (1997).

44. Q. Huang, A. Santoro, J. W. Lynn, R. W. Erwin, J. A. Borchers, J. L. Peng, K. Ghosh, and R. L. Greene, Phys. Rev. B58, 2684 (1998).

45. L. M. Rodriguez-Martinez and J. P. Attfield, Chem. Mater. 11, 1504 (1999).

46. S. G. Hibble, S. P. Cooper, A.C. Hannon, I. D. Fawcett, and M. Greenblatt, J. Phys.: Condens. Matter 11, 9221 (1999).

47. A. Mellergard, R. L. McGreevy, and S. G. Eriksson, J. Phys.: Condens. Matter 12, 4975 (2000).

48. V. Caignaert, E. Suard, A. Maignan, C. Simon, and B. Raveau, C. R. Acad. Sci. Paris 321, 515 (1995).

49. M. R. Ibarra, P. A. Algarabel, C. Marquina, J. Blasco, and J. Garsia, Phys. Rev. Lett. 75, 3541 (1995).

50. S. E. Lofland, P. Kim, P. Dahiroe, S. M. Bhagat, S. D. Tyagi, S. G. Karabshev, D. A. Shulyatev, A. A. Arsenov, and Y. Mukovskii, Phys. Lett. A233, 476 (1996).

51. Cz. Kapusta, P. C. Riedi, W. Kocemba, G. J. Tomka, M. R. Ibarra, J. M. De Teresa, M. Viret, and J. M. D. Coey, J. Phys.: Condens. Matter 11, 4079 (1999).

52. P. Dai, J. A. Fernandez-Baca, N. Wakabayashi, E. W. Plummer, Y. Tomioka, and Y. Tokura, Phys. Rev. Lett. 85, 2553 (2000).

53. C. P. Adams, J. W. Lynn, Y. M. Mukovskii, A. A. Arsenov, and D. A. Shulyatev, Phys. Rev. Lett. 85, 3954 (2000).

54. V. Chechersky, A. Nath, M. A. Subramanian, and R. L. Greene, unpublished work.

55. O. Lang, C. Felser, R. Seshadri, F. Renz, J.-M. Kiat, J. Ensling, P. Gutlich, and W. Tremel, Adv. Mater. 12, 65 (2000).

56. A. Nath, Z. Klencsar, E. Kuzmann, Z. Homonnay, A. Vertes, A. Simopoulos, E. Devlin, G. Kallias, A. P. Ramirez, and R. J. Cava, unpublished work. 